\title{
CDISC CDASH Concomitant Medication Route of Administration Terminology
}

National Cancer Institute

\section{Source}

National Cancer Institute. CDISC CDASH Concomitant Medication Route of

Administration Terminology. NCI Thesaurus. Code C78420.

A terminology subset of the CDISC Study Data Tabulation Model (SDT M) Route codelist created for Clinical Data Acquisition Standards Harmonization (CDASH) Concomitant Medication Route of Administration terminology. 